\title{
EPR Line Shifts and Line Shape Changes Due to Spin Exchange of Nitroxide Free Radicals in Liquids 2. Extension to High Spin Exchange Frequencies and Inhomogeneously Broadened Spectra
}

\author{
Barney L. Bales* and Miroslav Peric \\ Department of Physics and Astronomy and The Center for Supramolecular Studies, \\ California State University at Northridge, Northridge, California 91330
}

Received: December 13, 2001; In Final Form: February 21, 2002

\begin{abstract}
The work in part 1 of this series (J. Phys. Chem. B. 1997, 101, 8707) is extended experimentally and theoretically to include inhomogeneously broadened nitroxide spectra and high spin exchange frequencies. The nitroxide spin probe 16-doxylstearic acid methyl ester, which is severely inhomogeneously broadened by unresolved hyperfine structure in the absence of spin exchange, is studied under conditions in which the spin probe undergoes spin exchange varying from slow exchange to high exchange leading to collapse of the three ${ }^{14} \mathrm{~N}$ hyperfine lines into a single line. Over the entire spin exchange frequency range, theoretically predicted spectra are described essentially perfectly by the sum of three absorption and two "dispersion" lines of Lorentzian shape. The dispersion components are of opposite signs for the outer lines and zero for the inner. The intensities and the line widths of the outer absorption lines are different from the center line. Theoretically, adding unresolved hyperfine structure which inhomogeneously broadens the lines, has no effect on the line widths or intensities of the absorption lines, the intensity of the dispersion lines, or the line shifts. Comparing theory with experiment shows that the line widths, intensities, and line shapes are in excellent accord, whereas the line shifts are not; experimental lines move toward one another faster than predicted by theory.
\end{abstract}

\section{Introduction}

In part $1,{ }^{1}$ we derived an expression for the EPR spectrum of a nitroxide free radical undergoing spin exchange in the slow exchange limit following the perturbation theory developed by Molin et al. $^{2}$ and showed that, in the slow exchange limit, experimentally observed line shapes from the nitroxide spin probe peroxylamine disulfonate (PADS) were in accord with the perturbation theory. PADS is the simplest of all nitroxide probes because there are no interacting protons leading to inhomogeneous broadening of the lines. Here, we extend the investigation to a spin probe severely broadened inhomogeneously by unresolved proton hyperfine structure and extend the range of spin exchange frequencies. The extension to inhomogeneously broadened spectra is important because virtually all nitroxide spin probes belong to this class. Inhomogeneous broadening is conveniently expressed as the Gaussian component of a Voigt line shape. ${ }^{3}$ Perdeuteration of spin probes can reduce this Gaussian component ${ }^{3}$; however, for many spin probes, especially those that tend to be used in biological studies, it is still not negligible. ${ }^{3}$ The extension to high spin exchange frequencies could be important for both theoretical and practical reasons. Theoretically, at high spin exchange frequencies, the accepted theory of spin exchange may be tested and the extent to which the perturbation theory is applicable may be probed. Practically, there may be experimental cases in which high spin exchange is unavoidable in compartmentalized liquids such as micelles or in biological systems. In these cases, reducing the spin probe concentration to reduce the spin exchange frequency, which is a strategy that can be employed in normal solutions,

* To whom correspondence should be addressed. E-mail: barney. bales@csun.edu. Fax: (818) 677-3234. might not work because the presence of even two spin probes in a compartment could lead to high exchange.

For this study, we have selected the nitroxide spin probe 16doxylstearic acid methyl ester (16DSE) for two reasons. First, we have found ${ }^{4,5}$ this spin probe to be convenient for micelle research, combining the useful properties of being quite hydrophobic, thus partitioning strongly to the micelle pseudophase, and yet displaying EPR spectra in the fast motion regime yielding narrow EPR lines. Second, it is a spin probe severely inhomogeneously broadened by a hyperfine structure that is known in detail from NMR studies. ${ }^{6}$ In this work, we do not pursue the interesting question of the probability of spin exchange upon bimolecular collision. ${ }^{2}$ Perhaps some conformations of 16DSE would inhibit spin exchange during the lifetime of the collision pair. In this work, the details leading to spin exchange are unimportant; we vary the spin exchange frequency by varying the temperature or the concentration of the spin probe and find identical results.

We find that the line shapes predicted by perturbation theory can be applied to analyze spin exchange broadened spectra to as high a spin exchange as one wishes. We also find that the generally accepted theory is in agreement with experiment in every detail except one: the shift of the hyperfine lines is not properly predicted for spectra inhomogeneously broadened by unresolved hyperfine structure.

See part $1^{1}$ and the references therein for a discussion of history of spin exchange and the motivations behind studying the phenomenon again after a relatively quiet period for about 25 years in which little work has been done on the subject. We briefly reiterate the important new findings. Spin exchange frequencies of high precision may be found in three ways: line broadening, line shifts, and the intensities of two spin-exchange 
induced dispersion lines. Thus, compared with the traditional method of studying spin exchange from line broadening alone, significantly more information is available. Part $1^{1}$ showed that all three of these sources of information gave spin exchange frequencies that were in agreement with each other and with theory for PADS which exhibits ${ }^{14} \mathrm{~N}$ hyperfine structure only. Part $1^{1}$ showed that the perturbation theory yielded rather accurate results to spin exchange frequencies much higher than those contemplated in the perturbation theory. Here, we show that line broadening and dispersion intensities yield accurate spin exchange frequencies as predicted by theory to quite high spin exchange frequencies. The results from line shifts could also yield accurate spin exchange frequencies; however, because these are somewhat different than those predicted by the accepted theory, they would have to be on an empirical basis for the moment.

\section{Theory}

The generally accepted expression ${ }^{2,7}$ describing the line shape is given by the real part of eq 1

$$
G(H)=\frac{S(H)}{1-\frac{\omega_{e}}{\gamma} S(H)}
$$

where $H$ is the magnetic field, $\omega_{\mathrm{e}}$ is the spin exchange frequency, $\gamma$ is the gyromagnetic ratio of the electron, and $S(H)$ is given by

$$
S(H)=\sum \frac{\rho_{j}}{i\left(H-H_{j}\right)+\gamma^{-1}\left[\omega_{e}+\left(T_{2}^{-1}\right)_{j}\right]}
$$

In eq $2, i=\sqrt{-1}$ and the sum is over $j$, which denotes the jth resonance line in the spectrum which appears at resonance field $H_{j}$, with degeneracy $\rho_{j}$, and is characterized by spin-spin relaxation time $\left(T_{2}\right)_{\mathrm{j}}$. The peak-to-peak first-derivative Lorentzian line width of the jth line at $\omega_{\mathrm{e}}=0$ is given by $\Delta H_{\mathrm{pp}}^{\mathrm{L}}$ $(0)_{j}=2 / \sqrt{3} \gamma T_{2 j}$. Each line in the sum of eq 2 is assumed to be of Lorentzian shape. We call eq 1 the "rigorous theory".

Molin et al. ${ }^{2}$ applied second-order perturbation theory to eq 1 in the slow exchange limit where $\omega_{\text {ex }} / \gamma$ was small compared with the hyperfine spacings. In part $1,{ }^{1}$ we applied their theory to the case of a nitroxide spin probe having hyperfine spacing in the absence of spin exchange $\mathrm{A}_{0}$. See part $1^{1}$ for details. Briefly, for nitroxide free radicals tumbling rapidly, producing motionally narrowed EPR spectra in the absence of unresolved hyperfine structure, the resonance lines have the Lorentzian shape, which are broadened by spin exchange by

$$
<B>=\frac{4 \omega_{\mathrm{e}}}{3 \sqrt{3} \gamma}
$$

where the broadening, $\langle B>$ is the average value of the broadening of the three lines, $B_{\mathrm{M}_{\mathrm{I}}}$, defined to be

$$
B_{\mathrm{M}_{\mathrm{I}}}=\Delta H_{\mathrm{pp}}^{\mathrm{L}}\left(\omega_{\mathrm{e}}\right)_{M_{\mathrm{I}}}-\Delta H_{\mathrm{pp}}^{\mathrm{L}}(0)_{M_{\mathrm{I}}}
$$

That is

$$
<B>=\frac{1}{3}\left(B_{+1}+B_{0}+B_{-1}\right)
$$

The subscript $M_{\mathrm{I}}=+1,0$, and -1 labels the hyperfine lines at low-, central- and high-fields, respectively.
Previously ${ }^{1}$, the line shapes for PADS were accurately Lorentzian, so a straightforward measurement was required to evaluate $B_{\mathrm{M}_{\mathrm{I}}}$. For the nitroxide probe considered here, 16doxylstearic acid methyl ester (16DSE) the line width is dominated by unresolved hyperfine structure in the absence of spin exchange. Thus, for this work, we are required to separate the spin relaxation induced component of the line width (Lorentzian) from the component produced by unresolved hyperfine structure (Gaussian) for low values of $\omega_{\mathrm{e}}$. This separation is achieved by fitting the lines to an approximate Voigt shape which is now a routine procedure, ${ }^{3,8}$ thus presenting no problems.

Experimentally, $\omega_{\mathrm{e}}$ may be controlled by exploiting the fact that $\omega_{\mathrm{e}}$ is proportional to the concentration, thus

$$
\omega_{\mathrm{e}}=K[16 \mathrm{DSE}]
$$

where [16DSE] is the molar concentration of 16DSE and the factor $K$ depends on the temperature, viscosity, charge of the nitroxide, ionic strength, ${ }^{9}$ and perhaps steric factors ${ }^{2}$. The value of $\omega_{\mathrm{e}}$ may also be varied by changing the temperature, and therefore the viscosity of the solution. Often, the StokesEinstein relation holds

$$
K=\text { constant } \bullet T / \eta
$$

where $T$ is the absolute temperature, and $\eta$ is the shear viscosity; however, this is not necessary to reach the conclusions of this work.

Previously, we showed that the perturbation theory ${ }^{2}$, in firstderivative field-swept form, yielded a spectrum that could be written in terms of the peak-to-peak heights of the absorption component, $V_{\mathrm{pp}}\left(M_{\mathrm{I}}\right)$, and the maximum amplitudes, $V_{\text {disp }}\left(M_{\mathrm{I}}\right)$, (positive for $M_{\mathrm{I}}=+1$ and negative for $M_{\mathrm{I}}=-1$ ) of the spinexchange dispersion component as follows

$$
Y^{\prime}\left(H, \omega_{\mathrm{ex}}\right)=\sum_{M_{\mathrm{I}}}\left[V_{\mathrm{PP}}\left(M_{I}\right) L_{M_{\mathrm{I}}}^{\prime}(H)+V_{\mathrm{disp}}\left(M_{\mathrm{I}}\right) D_{M_{\mathrm{I}}}^{\prime}(H)\right]
$$

In eq $8, H$ denotes the value of the magnetic field which is swept and $L_{M_{\mathrm{I}}}^{\prime}(H)$ is the Lorentzian first-derivative absorption of unit peak-to-peak height given by

$$
L_{M_{\mathrm{I}}}^{\prime}(H)=\frac{-8 \xi_{M_{\mathrm{I}}}^{\prime}}{\left[3+\xi_{M_{\mathrm{I}}}^{\prime 2}\right]^{2}}
$$

In eq 8 , the spin-exchanged induced dispersion of unit maximum amplitude is given by

$$
D_{M_{\mathrm{I}}}^{\prime}(H)=\frac{3\left[3-\xi_{M_{\mathrm{I}}^{\prime}}^{2}\right]}{\left[3+\xi_{M_{\mathrm{I}}}^{2}\right]^{2}}
$$

In eqs 9 and $10, \xi_{M_{\mathrm{I}}}^{\prime}$ is defined by

$$
\xi_{M_{\mathrm{I}}}^{\prime}=2 \frac{H-H\left(\omega_{\mathrm{ex}}\right)_{M_{\mathrm{I}}}}{\Delta \mathrm{H}_{\mathrm{pp}}^{L}\left(\omega_{\mathrm{ex}}\right)_{M_{\mathrm{I}}}}
$$

where $H\left(\omega_{\mathrm{ex}}\right)_{M_{\mathrm{I}}}$ is the resonance field of the $\mathrm{M}_{\mathrm{I}}$ line under the influence of spin exchange. To simplify the presentation, we use the term "dispersion" to mean the spin-exchanged induced dispersion terms in eq 8 .

The perturbation theoretical prediction of the ratio of the dispersion and absorption heights was shown ${ }^{1}$ to be 


$$
V_{\mathrm{disp}}\left(M_{\mathrm{I}}\right) / V_{\mathrm{pp}}\left(M_{\mathrm{I}}\right)=M_{\mathrm{I}} \frac{4 \omega_{\mathrm{ex}}}{3 \sqrt{3} \gamma A_{0}}
$$

which may be written in terms of $\langle B>$ using eq 3 as follows

$$
V_{\text {disp }}\left(M_{\mathrm{I}}\right) / V_{\mathrm{pp}}\left(M_{\mathrm{I}}\right)=M_{\mathrm{I}} \frac{<B>}{A_{0}}
$$

Note that eq 13 supposes that the broadening $\langle\mathrm{B}\rangle$ is due to spin exchange and not some other concentration-dependent broadening. Note that the factor $M_{\mathrm{I}}$ on the right-hand side of eq 13 is due to the fact that $V_{\text {disp }}\left(M_{\mathrm{I}}\right)$ is positive for $M_{\mathrm{I}}=+1$ and negative for $M_{\mathrm{I}}=-1$.

There are two shifts of hyperfine lines that entered the perturbation theory. The first, directly due to spin exchange, shifts the resonance frequencies of the outer two lines toward the center, resulting in a hyperfine spacing as follows

$$
A_{\mathrm{abs}}=\frac{1}{2}\left\{H\left(\omega_{\mathrm{ex}}\right)_{-1}-H\left(\omega_{\mathrm{ex}}\right)_{+1}\right\}
$$

which, according to the perturbation theory ${ }^{1}$ is given by

$$
A_{\mathrm{abs}}=A_{0}-\frac{1}{6 A_{0}}\left(\omega_{\mathrm{ex}} / \gamma\right)^{2}
$$

The subscript abs refers to the absorption lines. In addition to the shift leading to the spacing $A_{\mathrm{abs}}$, eq 14, there is also a shift due to the overlap of the dispersion lines pushing the outer lines further toward the center. This results in a hyperfine spacing, $A_{\mathrm{obs}}$, defined as one-half the difference in the fields where the low- and high-field lines cross the baseline. The subscript $o b s$ refers to the observed spacing. $A_{\mathrm{obs}}$ is directly measurable from the EPR spectrum; $A_{\mathrm{abs}}$ must be found by fitting of eq 8 to the experimental spectrum. See part 1 for the perturbation theory prediction for $A_{\mathrm{obs}}$. For data in the slow exchange limit, $A_{\mathrm{obs}}$ is a more useful parameter because it is about a factor of 3 more sensitive to spin exchange ${ }^{1}$ than $A_{\text {abs }}$; however, it is less fundamentally related to the theory than $A_{\mathrm{abs}}$. Further, as the spin exchange frequency increases, $A_{\mathrm{obs}}$, becomes undefined (see Figures 4 and 5). Therefore, in the remainder of this paper, we deal exclusively with $A_{\text {abs }}$.

The shift in eq 15 may be written in terms of the broadening by employing eq 3

$$
A_{\mathrm{abs}} / A_{0}=1-\frac{9}{32}\left(\frac{<B>}{A_{0}}\right)^{2}
$$

The spectrum in Figure 1a is computed from eq 1 for a value of $\left\langle B>/ A_{0}=0.2273\right.$. The following two traces show the best fit of the computed spectrum to three absorption, Figure $1 b$, and two dispersion lines, Figure 1c, of Lorentzian shape, eq 8. The quantities that appear in eqs 13 and 14 are defined in Figure 1.

The doubly integrated intensity of each absorption line, $I_{\mathrm{MI}}$, may be calculated from the following ${ }^{1}$, valid for Lorentzian line shapes

$$
V_{\mathrm{PP}}\left(M_{\mathrm{I}}\right)=\sqrt{3} I_{M_{\mathrm{I}}} / \pi\left[\Delta H_{\mathrm{pp}}^{\mathrm{L}}\left(\omega_{\mathrm{ex}}\right)_{M_{\mathrm{I}}}\right]^{2}
$$

In the slow exchange limit, the perturbation theory predicts that the broadening is the same for all three lines and that the doubly integrated intensities of the three lines are equal to one another, independent of $\omega_{\mathrm{ex}}$. If we constrain the fits of the spectra computed from the rigorous theory, eq 1 , to eq 8 employing

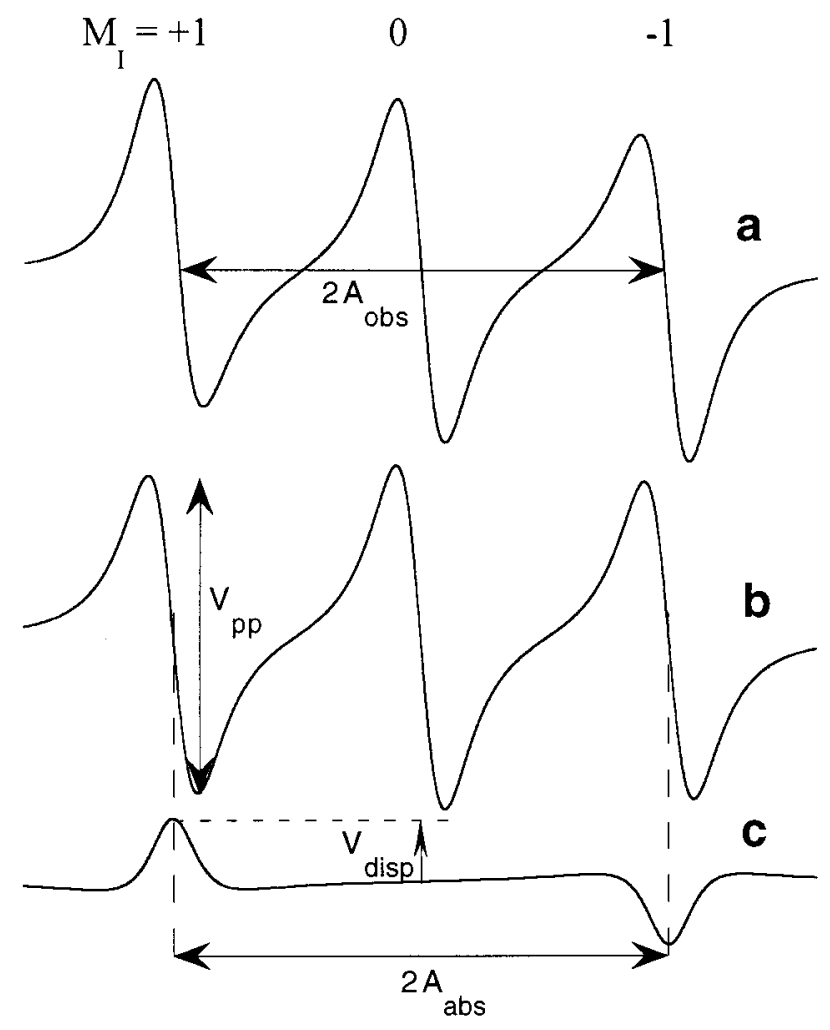

Figure 1. EPR spectrum of a nitroxide radical undergoing spin exchange at frequency $\omega_{\mathrm{ex}} / \gamma A_{0}=0.29533$ calculated from the rigorous theory, eq 1 (a). Three absorption lines (b) and two dispersion lines (c) in eq 8 adjusted in intensity and line width to least-squares fit the rigorous theory. The sum of the five lines in (b) and (c) is exactly equal to (a) if the width and intensity of the center line are allowed to be different than the outer lines in the least-squares fitting. The characteristic "sloping" of a spin exchanged broadened spectrum down and to the right in (a) is due to overlap of the two dispersion lines of opposite sign in (c). The parameters in eqs 13 and 14 are defined.

these two criteria, the fits get progressively worse as $\left\langle B>/ A_{0}\right.$ increases. If we relax both, the fits are excellent for all values of $\langle B\rangle / A_{0}$ including those at high values which lead to the collapse of the three hyperfine lines into a single line. In other words, the rigorous theory may be represented by eq 8 defined by the parameters $V_{\mathrm{pp}}\left(M_{\mathrm{I}}\right), V_{\mathrm{disp}}\left(M_{\mathrm{I}}\right), H\left(\omega_{\mathrm{ex}}\right)_{M_{\mathrm{I}}}$ and $\Delta H_{\mathrm{pp}}^{\mathrm{L}}\left(\omega_{\mathrm{ex}}\right)_{M_{\mathrm{I}}}$.

\section{Materials and Methods}

16DSE and reagent grade ethanol were purchased from Sigma and used as received. A mother solution of 16DSE of concentration $51.7 \pm 1.5 \mathrm{mM}$ was prepared by weight in ethanol using the known density of ethanol. The mother solution was deoxygenated by bubbling $\mathrm{N}_{2}$ gas for one minute through a 50 $\mu \mathrm{L}$ disposable pipet. This is double the recommended time for deoxygenation as presented by Jost and Griffith. ${ }^{10}$ A sample of the mother solution was immediately sealed into this same, flushed pipet. The remaining solution was weighed and more ethanol was added. This procedure was repeated resulting in the concentrations given in Table 1. Intermediate weighings controlled the amount of solvent lost to evaporation. The concentrations are estimated to be accurate to about $3 \%$ and the relative concentrations accurate to about one-half this value.

All of the samples in Table 1 were run at three temperatures: $25.7,-14$, and $6.4^{\circ} \mathrm{C}$. Samples A, D, and K were run from $-25^{\circ} \mathrm{C}$ to $76^{\circ} \mathrm{C}$. This is a large amount of data; too much to effectively present in figures. Therefore, we have selected for presentation data for all samples run at 6.4 and $25.7^{\circ} \mathrm{C}$ and data for sample $\mathrm{A}$ as a function of temperature. All data above 


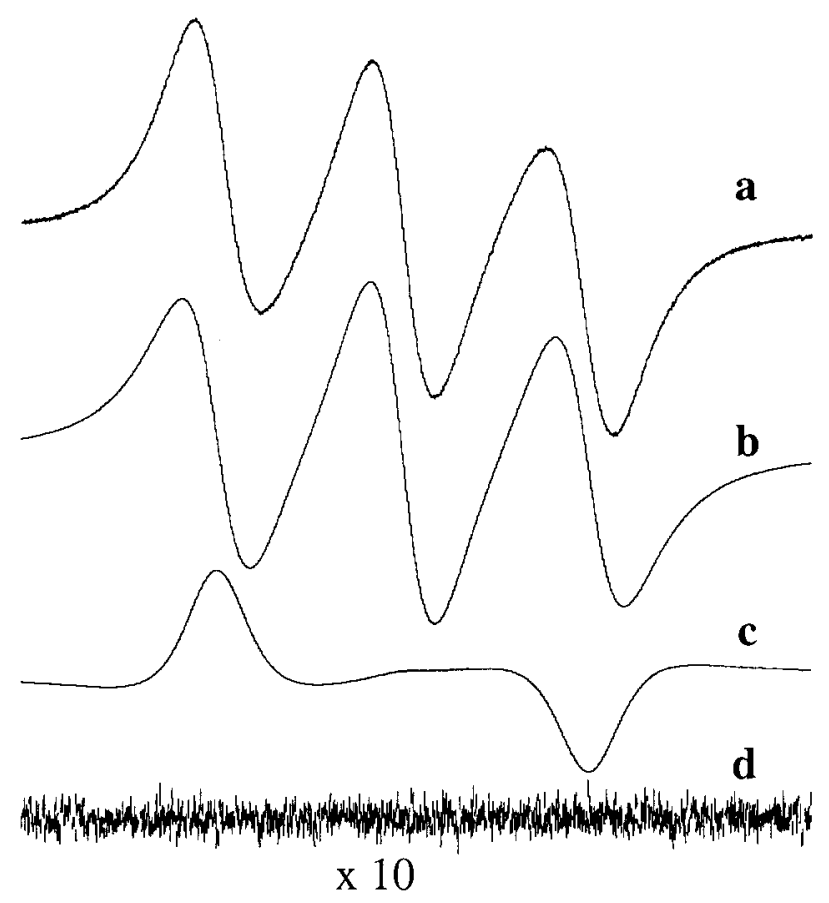

Figure 2. Experimental spectrum of $51.7 \mathrm{mM}$ 16DSE in EtOH at $T$ $=25.7^{\circ} \mathrm{C}$ (a). Absorption (b) and dispersion lines (c) in eq 8, leastsquares fit to the experimental spectrum. Careful inspection of (c) shows a small dispersion line due to instrumental dispersion, which is subtracted from the outer two lines to correct the values of $V_{\text {disp }}( \pm 1)$. The difference in the experimental spectrum and the fit multiplied by 10 is shown in (d). This spectrum is broadened by $\langle B\rangle=5.746 \pm$ $0.001 \mathrm{G}$ and the lines are shifted by $A_{\mathrm{abs}} / A_{0}=0.9254 \pm 0.0001$, where the uncertainties are the standard deviations in five measurements.

$-14{ }^{\circ} \mathrm{C}$ are within experimental error of those presented. There is no evidence for self-association of 16DSE at any temperature even at the highest concentration. Such association would be easily detected as a broad single line. ${ }^{11}$ The doubly integrated intensity was accurately proportional to the spin probe intensity, another indication that self-association did not occur. We note that even if self-association were to occur and to go undetected, this would not affect the results because such association would effectively reduce the spin probe concentration, the value of which is not needed in this work.

A thermocouple was placed above the sample in configuration $\mathrm{C}$ of Figure 1 of ref. ${ }^{12}$ After correction for the temperature gradient, ${ }^{12}$ the sample temperature is estimated to be accurate to $\pm 1{ }^{\circ} \mathrm{C}$. Neither the concentration nor the temperature is a critical parameter in this work because both are removed as parameters in eqs 13 and 16. For this reason, concentrations were not corrected for density changes with temperature.

EPR spectra were measured with a Bruker 300 ESP X-band spectrometer interfaced with Bruker's computer. Spectra were acquired using a sweep time of $21 \mathrm{~s}$; microwave power, $5 \mathrm{~mW}$; time constant, $5 \mathrm{~ms}$; sweep width, $60 \mathrm{G}$; modulation amplitude $0.23 \mathrm{G}$. This modulation amplitude broadens the Gaussian component of the line width by $0.009 \mathrm{G}$. After correcting for this broadening, ${ }^{13} \Delta H_{\mathrm{pp}}^{\mathrm{G}}=0.854 \pm 0.005 \mathrm{G}$ at $23.4{ }^{\circ} \mathrm{C}$ in good agreement with previous NMR and EPR determinations. ${ }^{6}$ The Gaussian line width is slightly temperature dependent. For example, $\Delta H_{\mathrm{pp}}^{\mathrm{G}}=0.872 \pm 0.001$ at $T=6.4^{\circ} \mathrm{C}$ and $\Delta H_{\mathrm{pp}}^{\mathrm{G}}=$ $0.896 \pm 0.011$ at $T=-13.9^{\circ} \mathrm{C}$. The sweep width was measured by Bruker's NMR Gaussmeter operating in the $1 \mathrm{mG}$ resolution mode and was averaged over the entire experiment.

Two sets of calculations of eq 1 were carried out on a personal computer using the equations from the Appendix of ref 1 . The

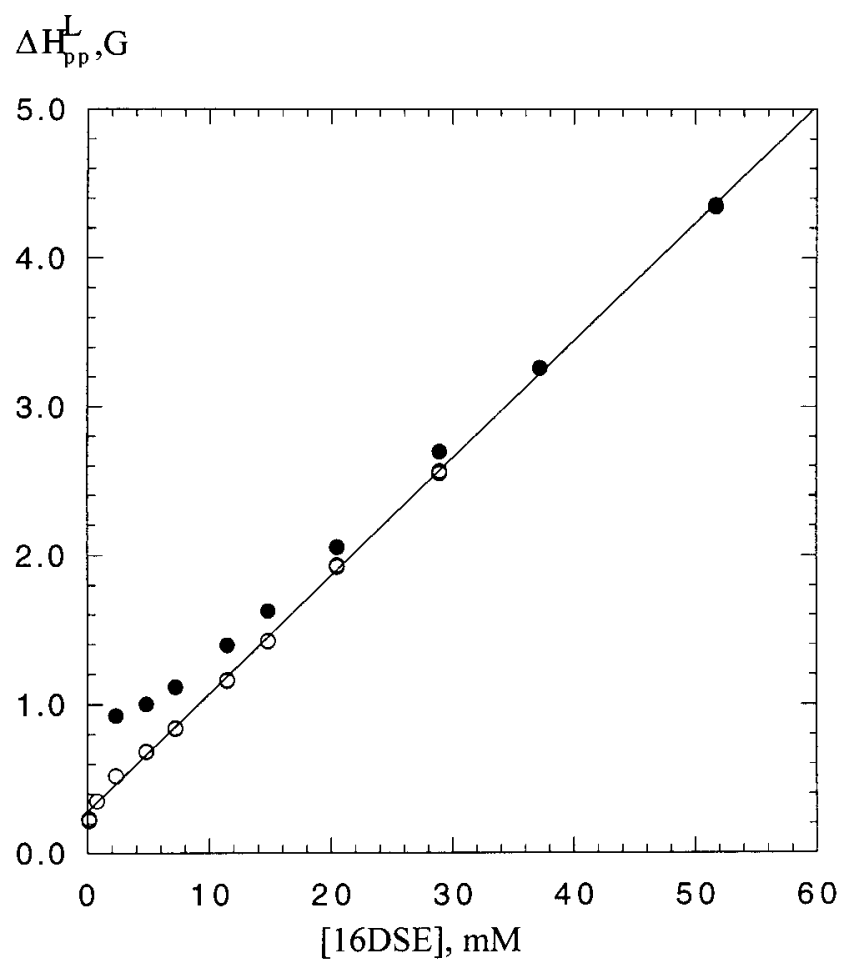

Figure 3. Example of the determination of the Lorentzian line width by fitting to a Voigt shape without dispersion, open circles, and the Lorentzian shape, with both absorption and dispersion, eq 8 , closed circles. The concentration of 16DSE is varied at $T=6.4{ }^{\circ} \mathrm{C}$ in EtOH. At low spin exchange frequencies, the Lorentzian shape in eq 8 yields inaccurate results and at high spin exchange frequencies, the neglect of line overlap in a Voigt leads to poor fits. In all cases, there is an intermediate range of spin exchange frequencies, at overall line widths between 1.8 and $2.5 \mathrm{G}$, where the two approaches agree; in this case at 20.5 and $28.9 \mathrm{mM}$. Each symbol is actually 5 points from 5 separate spectra illustrating the reproducibility of the measurements.

first set corresponded to a nitroxide with no inhomogeneous broadening; i.e., three hyperfine lines separated by $A_{0}-$ $A_{0}^{2} / 2 H(0)_{0}$ between the $M_{\mathrm{I}}=+1$ and 0 lines and $A_{0}+$ $A_{0}^{2} / 2 H(0)_{0}$ between the $M_{\mathrm{I}}=0$ and -1 lines. The results expressed in normalized form; i.e., as $\langle B\rangle / A_{0}$ and $A_{\text {abs }} / A_{0}$, are independent of the chosen values of $A_{0}$; most of the present calculations employed $A_{0}=14.8000 \mathrm{G}$. The small second-order shift ${ }^{14} A_{0}^{2} / 2 H(0)_{0}$ had a negligible effect on the results. The second set corresponded to a nitroxide with inhomogeneous broadening designed to match $\Delta H_{\mathrm{pp}}^{\mathrm{G}}=0.854 \mathrm{G}$ as follows: each of the three ${ }^{14} \mathrm{~N}$ hyperfine lines were split into a quintet of relative intensity $1: 4: 6: 4: 1$ of spacing $a_{\mathrm{p}}=0.3771 \mathrm{G}$ due to hyperfine interaction with $n=4$ equivalent protons. Thus the sums in eq 1 are over 15 lines. The value of $\Delta H_{\mathrm{pp}}^{\mathrm{G}}$ is computed from the following ${ }^{3}$

$$
\Delta H_{\mathrm{pp}}^{\mathrm{G}}=\sqrt{\alpha n} a_{\mathrm{p}}
$$

where $\alpha=1.282$ is appropriate to $n=4$ s see Table 2 of ref $13\}$. We refer to these two sets of calculations as the homogeneous and inhomogeneous theoretical spectra. In the figures, the homogeneous case is shown with a solid line, whereas the inhomogeneous case is indicated with small, filled circles. The use of a simplified hyperfine pattern to study inhomogeneous broadening is justified by the fact that once a hyperfine multliplet becomes unresolved, the details of the hyperfine pattern are unimportant at $\omega_{\mathrm{e}}=0^{3}$ and by the fact, shown below, that inhomogeneous broadening has no affect on the results from eq 1 to very high values of $\omega_{\mathrm{e}}$. 


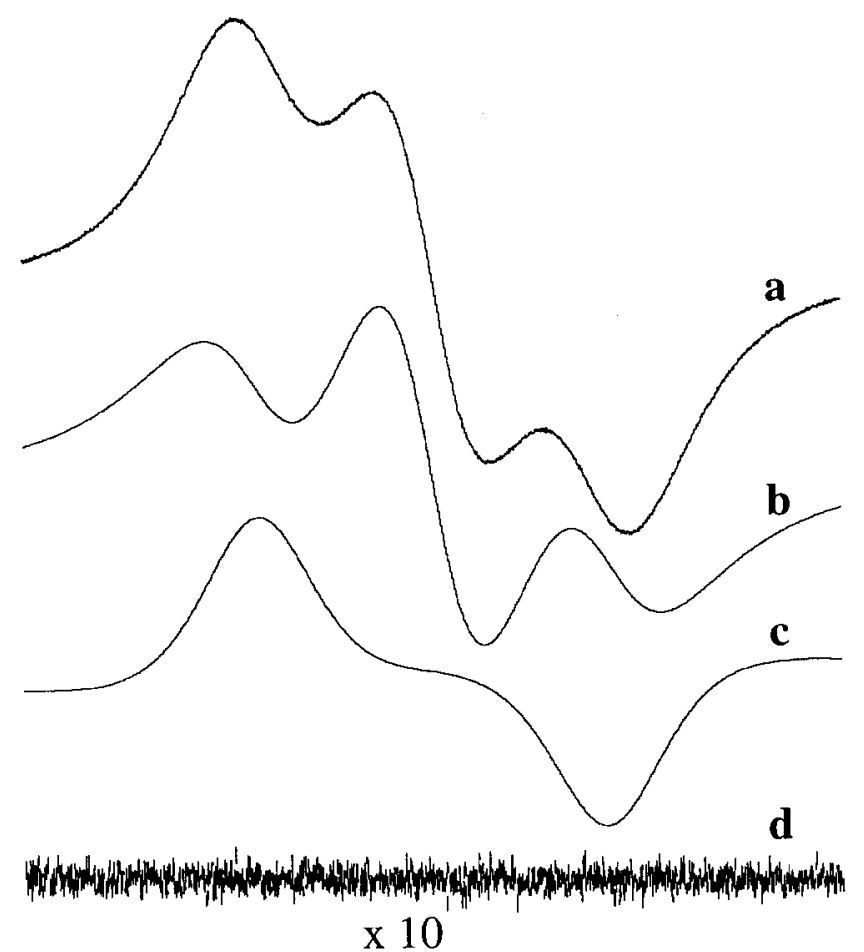

Figure 4. Experimental spectrum of $51.7 \mathrm{mM}$ 16DSE in EtOH at $T$ $=50{ }^{\circ} \mathrm{C}$, (a). Absorption (b) and dispersion lines (c) in eq 8, leastsquares fit to the experimental spectrum. The difference multiplied by 10 (d). This spectrum is broadened by $\langle B\rangle=8.111 \pm 0.003 \mathrm{G}$ and the lines are shifted by $A_{\text {abs }} / A_{0}=0.8603 \pm 0.0003$ where the uncertainties are the standard deviations in five measurements.

\section{Results and Discussion}

Figure 2a shows an experimental spectrum of $51.7 \mathrm{mM}$ 16DSE undergoing spin exchange in EtOH at $T=25.7{ }^{\circ} \mathrm{C}$. The spectrum in Figure 2a shows a pronounced "sloping" down and to the right characteristic of spectra under the influence of spin exchange. The fits to eq 8 are shown in Figure $2 b$ and $2 c$. The residuals, amplified by a factor of 10, are shown in Figure $2 \mathrm{~d}$ demonstrating that the fits are excellent indeed. The fits were carried out allowing the broadening to vary with $M_{\mathrm{I}}$ using as fit parameters the amplitudes $V_{\text {disp }}\left(M_{\mathrm{I}}\right), V_{\mathrm{pp}}\left(M_{\mathrm{I}}\right)$, the line positions of the absorption lines, $H\left(\omega_{\mathrm{ex}}\right)_{M_{\mathrm{I}}}$, and the Lorentzian line widths, $\Delta H_{\mathrm{pp}}^{\mathrm{L}}\left(\omega_{\mathrm{e}}\right)_{M_{\mathrm{I}}}$. From values of $H\left(\omega_{\mathrm{ex}}\right)_{M_{\mathrm{I}}}$, values of $A_{\mathrm{abs}}$ are computed from eq 14. Plotting these values of $A_{\text {abs }}$ versus [16DSE] at each temperature yields the intercept, $A_{0}$. Often, normal dispersion is observed in spectra obtained using lossy samples. This leads to a contribution to $V_{\text {disp }}\left(M_{\mathrm{I}}\right)$ that is of the same sign for all three lines which appears as a non zero value of $V_{\text {disp }}\left(M_{\mathrm{I}}\right)$ for the central line, $M_{\mathrm{I}}=0$. Careful inspection of the central part of Figure 2c shows an example of a minor contribution due to normal dispersion. Typical instrumental dispersion varied from 0 to $3 \%$. Instrumental dispersion was corrected $^{1}$ by subtracting $V_{\text {disp }}(0)\left\{\Delta H_{\mathrm{pp}}^{\mathrm{L}}\left(\omega_{\mathrm{e}}\right)_{0} / \Delta H_{\mathrm{pp}}^{\mathrm{L}}\left(\omega_{\mathrm{e}}\right)_{ \pm 1}\right\}^{2}$ from $V_{\text {disp }}(+1)$ and $V_{\text {disp }}(-1)$. The scaling factor proportional to the line width squared takes into account that line heights scale as the line width squared. Correction with or without the scaling were within experimental error of one another. An excellent test of the validity of this correction procedure is afforded by comparing $\left|V_{\text {disp }}(+1)\right|$ with $\left|V_{\text {disp }}(-1)\right|$, the values of which ought to equal.

All spectra were recorded five times, one after another. Representative results are presented graphically in Figures 3 and $7-10$ plotting all five points to demonstrate the reproducibility of the data.

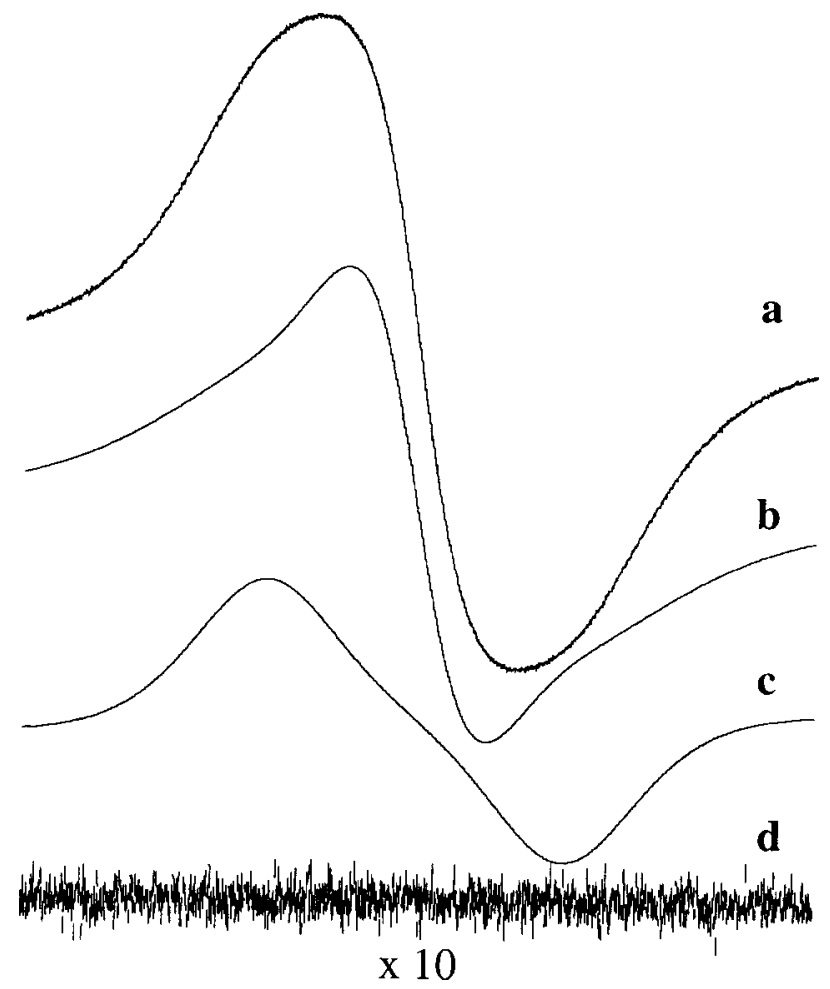

Figure 5. Experimental spectrum of $51.7 \mathrm{mM}$ 16DSE in EtOH at $T$ $=76^{\circ} \mathrm{C}$, (a). Absorption (b) and dispersion lines (c) in eq 8, leastsquares fit to the experimental spectrum. The difference multiplied by 10 (d). The severe overlap of the three absorption lines in (b) produces what appears to be a single line with shoulders. This spectrum is broadened by $\langle B>=10.89 \pm 0.071 \mathrm{G}$ and the lines are shifted by $A_{\text {abs }} / A_{0}=0.761 \pm 0.002$, where the uncertainties are the standard deviations in five measurements.

TABLE 1: 16DSE Concentrations ${ }^{a}$

\begin{tabular}{cccc}
\hline sample & {$[16 \mathrm{DSE}], \mathrm{mM}$} & sample & [16DSE], mM \\
\hline A & 51.7 & $\mathrm{G}$ & 7.17 \\
B & 37.2 & $\mathrm{H}$ & 4.78 \\
C & 28.9 & $\mathrm{I}$ & 2.30 \\
D & 20.5 & $\mathrm{~J}$ & 0.754 \\
E & 14.8 & $\mathrm{~K}$ & 0.0905 \\
F & 11.45 & &
\end{tabular}

${ }^{a}$ Estimated accuracy $\pm 3 \%$, relative uncertainty $\pm 1.5 \%$.

Fitting the experimental spectra to eq 8, which supposes Lorentzian line shapes, gives excellent results for values $\omega_{\mathrm{e}}$ high enough to broaden the lines by approximately $B=2 \mathrm{G}$ and above. For spin exchange frequencies lower that this, the values of $B$ are in error because the experimental line shapes are not well reproduced by the Lorentzian shape. Thus, for low spin exchange frequencies the spectra were also least-squares fit to a Voigt shape. ${ }^{3,8}$ Values of $\Delta H_{\mathrm{pp}}^{\mathrm{L}}\left(\omega_{\mathrm{e}}\right)_{M_{\mathrm{I}}}$ derived from fits to the Voigt shape but neglecting the dispersion term were found to be within experimental error of those fit with Voigt shapes for both the absorption and dispersion terms. This result may seem surprising at first; however, because the absorption and dispersion terms are orthogonal, the dispersion term does not affect the value of $\Delta H_{\mathrm{pp}}^{\mathrm{L}}\left(\omega_{\mathrm{e}}\right)_{M_{\mathrm{I}}}$ significantly. This is a convenient result because the program LOWFIT that we have used for years may be used to evaluate the broadening up to a value of the Lorentzian line width $\Delta H_{\mathrm{pp}}^{\mathrm{L}}\left(\omega_{\mathrm{e}}\right)_{M_{\mathrm{I}}}=2.5 \mathrm{G}$. Above this value, LOWFIT does not work well because it does not account for line overlap from other ${ }^{14} \mathrm{~N}$ hyperfine components. Fortunately, the validity of fits from LOWFIT and eq 8 overlap in the range 2.0-2.5 G. This is illustrated in Figure 3, where the Lorentzian 


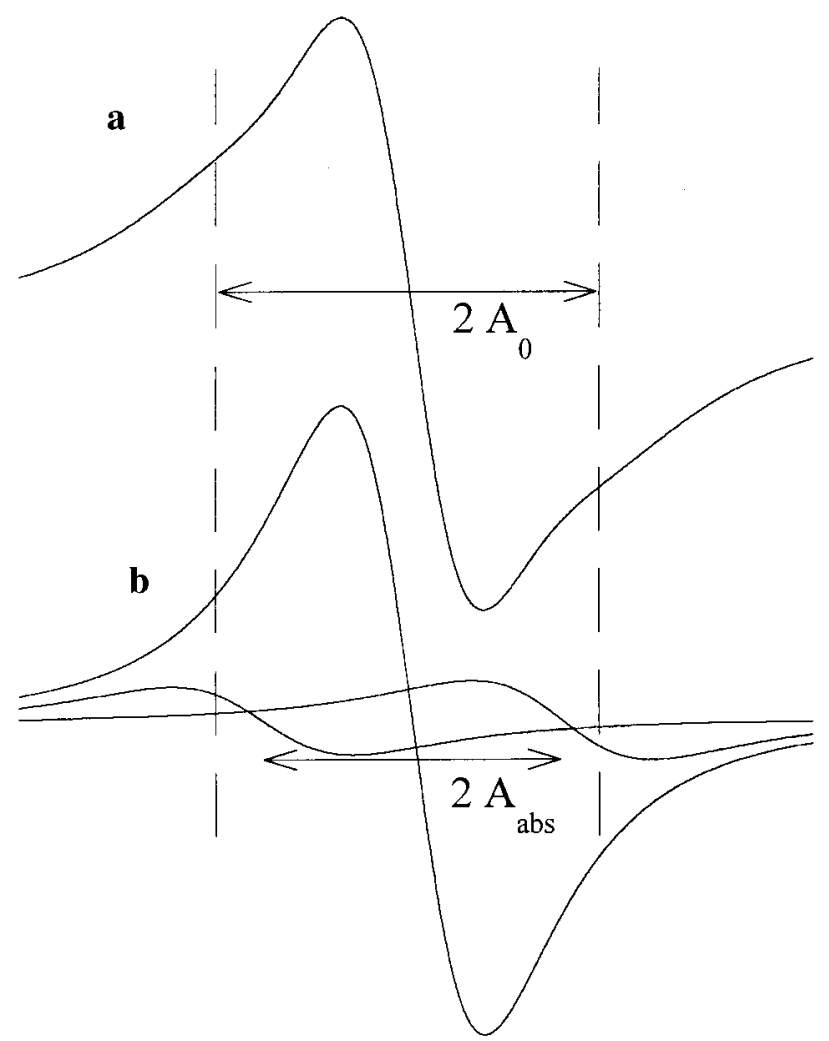

Figure 6. Sum of three overlapping Lorentzian absorption lines from Figure 5b (a). The three Lorentzian lines plotted separately (b). The vertical dashed lines fall at the positions of the lines in the absence of spin exchange, spaced by $2 A_{0}$. The inward shift of the outer lines by $A_{\text {abs }} / A_{0}=0.761 \pm 0.002$ and their loss of intensity to the center line are pronounced.

line width derived from fitting to a Voigt shape without dispersion, open circles, and fitting to eq 8, closed circles is shown for a run in which the concentration of 16DSE is varied at $T=6.4{ }^{\circ} \mathrm{C}$. Figure 3 is typical of all the temperatures and, in all cases, there is an intermediate range of spin exchange frequencies giving overall line widths in the range from 1.8 to $2.5 \mathrm{G}$, where the two approaches agree. In Figure 3, representing only one of the three hyperfine lines at one temperature, only two points lie in the range from 1.8 to $2.5 \mathrm{G}$; however, consider all three lines in all of the data collected, there were 150 such points. Below $1.8 \mathrm{G}$, the line width is determined from the fit to a Voigt; above $2.5 \mathrm{G}$ from the fit to eq 8 , and between, the average from the two methods. The average difference in the two methods in the range 1.8 to $2.5 \mathrm{G}$ is taken to be the uncertainty in the line width. The straight line is a linear leastsquares fit to the open circles alone, but is seen to pass accurately through the closed circles at higher spin exchange frequencies. The intercepts of such fits yield values of $\Delta H_{\mathrm{pp}}^{\mathrm{L}}(0)_{\mathrm{M}_{\mathrm{I}}}$; for example $\Delta H_{\mathrm{pp}}^{\mathrm{L}}(0)=0.22 \mathrm{G}$ in Figure 3 . Recalling that $\Delta H_{\mathrm{pp}}^{\mathrm{G}}$ is $0.87 \mathrm{G}$ at $6.4{ }^{\circ} \mathrm{C}$, the inhomogeneous broadening dominates the line width, being a factor of about four larger than the intrinsic line width. Values of $B_{M_{\mathrm{I}}}$ are then computed from eq 4 and their average values for a given spectrum from eq 5 .

The validity of the dual approach to measure $\Delta H_{\mathrm{pp}}^{\mathrm{L}}\left(\omega_{\mathrm{e}}\right)_{M_{\mathrm{I}}}$ employing a Voigt line shape at low values of $\omega_{\mathrm{e}}$ and eq 8 at high values of $\omega_{\mathrm{e}}$ was tested with inhomogeneously broadened spectra generated from eq 1. Curves similar to Figure 2 are produced with an overlap region where the results of the two approaches coincide. Computing $\langle B\rangle$ from these simulations verified the prediction of eq 3 . Including a dispersion term in the Voigt fits at low $\omega_{\mathrm{e}}$ did not improve the results.

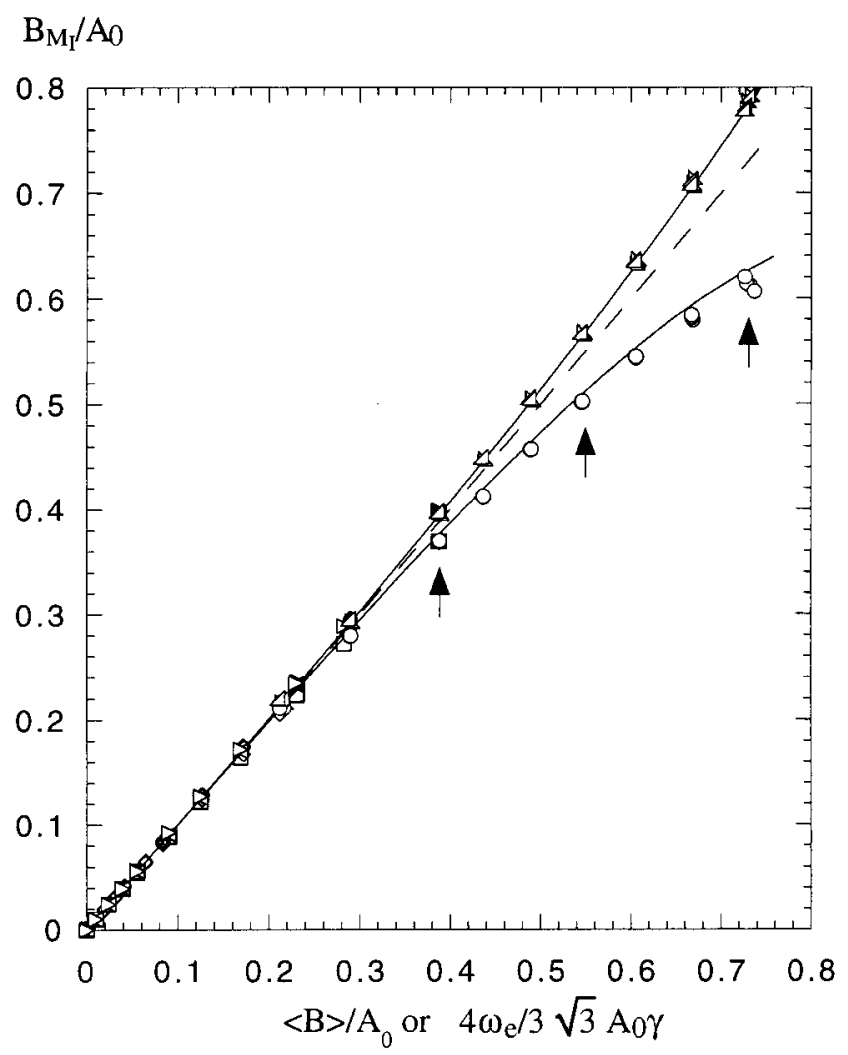

Figure 7. Broadening of the lines of 16DSE in EtOH varying [16DSE] at $6.4{ }^{\circ} \mathrm{C}$, for all three lines, (diamonds); varying [16DSE] at $25^{\circ} \mathrm{C}$, low-, (right isosceles triangles); center-, (squares); and high-field (left isosceles triangles) lines; varying the temperature for $51.7 \mathrm{mM}$ 16DSE, low-, (right right triangles); center-, (circles); and high-field (left right triangles) lines. The solid lines are derived from the rigorous theory, eq 1, whereas the dashed line is the average of the solid lines. The dashed line is also the prediction of the perturbation theory. The abscissa is the value of $\left\langle B>/ A_{0}\right.$ for the experimental points and $4 \omega_{\mathrm{e}} / 3 \sqrt{3} A_{0} \gamma$ for the theoretical. The theoretical curves have no adjustable parameters.

Figures 4 and 5 show spectra taken at progressively higher spin exchange frequencies together with the fits to eq 8 and the residuals displayed with a factor of 10 amplification. Clearly, in spectra such as those in Figures 4 and $5, A_{\text {obs }}$ is not defined since the outer lines do not cross the baseline. The least-squares fitting procedure relies on initial guesses at the parameters in eq $8 .^{8}$ For spectra such as those in Figures 2 and 4 , these initial guesses may be found from preliminary peak-picking routines; however, in spectra such as those in Figure 5 such guesses must be input by the user. The higher the value of $\omega_{\mathrm{e}}$, the more critical become the values of the initial guesses. With experience, the user learns to use initial guesses guided by the fits to a spectrum at a slightly lower value of $\omega_{\mathrm{e}}$. These remarks apply both to experimental spectra and theoretical spectra generated from eq 1.

Figure $5 b$ is indeed due to three overlapping Lorentzian lines although it appears to be a single line with shoulders. Figure $6 \mathrm{a}$ is the same as Figure $5 \mathrm{~b}$, whereas Figure $6 \mathrm{~b}$ displays the three lines separately; i.e., the three lines in Figure $6 \mathrm{~b}$ add up to Figure 5b. The dashed lines in Figure 6 are placed at the position of the outside lines in the absence of spin exchange; i.e., at a separation of $2 A_{0}$. Figure 6 dramatically illustrates the fact that the magnetization is transferred from the outer lines to the central line as spin exchange proceeds. The inward shift of the outer lines is $A_{\mathrm{abs}} / A_{0}=0.761 \pm 0.002$ where the uncertainty is the standard deviation in the measurement of five spectra. 
The outer lines are broadened faster than the central line as is evident from Figures 2, 4, and 6. Figure 7 shows the broadening of the individual lines as a function of the average line broadening for typical data. The vertical arrows correspond to the spectra in Figures 2, 4, and 5, respectively. Up to a value of $\left\langle B>/ A_{0}=0.3\right.$, all data, varying the concentration or the temperature, fall on the same $45^{\circ}$ line and the difference in broadening of the outer versus the inner lines is within experimental error. In Figure 7 , above $\left\langle B>/ A_{0}=0.3\right.$, the difference in the line widths of the central line (circles) and the outer lines (right and left right triangles) becomes apparent. The points corresponding to $M_{\mathrm{I}}=+1$ (right right triangles) and -1 (left right triangles) fall accurately on top of each other on the scale of Figure 7 demonstrating that the two outer lines are broadened equally and illustrates the level of precision that can be obtained in the measurement of line widths. The solid lines are derived from the rigorous theory, eq 1 , whereas the dashed line is the average of the solid lines. The dashed line is also the prediction of the perturbation theory. From these results, it is concluded that, for all values of $\omega_{\mathrm{e}}$, eq 3 holds provided one uses the average value of the broadening, $\langle B>$. The abscissa in Figure 7 is the value of $\left\langle B>/ A_{0}\right.$ for the experimental points and $4 \omega_{\mathrm{e}} / 3 \sqrt{3} A_{0} \gamma$ for the theoretical.

Figure 8 a shows a plot of the two values of $\left|V_{\text {disp }} / V_{\mathrm{pp}}\right|$ as a function of $\left\langle B>/ A_{0}\right.$. Various combinations of temperatures and concentrations are presented in Figure 8 as detailed in the figure caption. The solid line is derived from the rigorous theory, eq 1 , for homogeneously broadened spectra, whereas the solid circles that appear to fall on top of the solid line are computed from eq 1 using inhomogeneously broaden lines. The dashed line is the prediction of the perturbation theory, eq 13. Figure $8 \mathrm{~b}$ is an expanded presentation of (a) at lower values of spin exchange frequency, where the linear correlation of the values of $\left|V_{\text {disp }} / V_{\mathrm{pp}}\right|$ with $<B>/ A_{0}$ is evident. In Figure $8 \mathrm{~b}$, below values of $\langle B\rangle / A_{0}=0.05$, the experimental values are somewhat below the theoretical prediction. This discrepancy is not due to the use of the incorrect line shape in eq 8; the discrepancy persists when Voigt shapes are substituted for both the absorption and dispersion terms. Thus at low values of $\omega_{\mathrm{e}}$, the precision in determining $\omega_{\mathrm{e}}$ from $\left|V_{\mathrm{disp}} / V_{\mathrm{pp}}\right|$ is not as good for inhomogeneously broadened lines as it was previously ${ }^{1}$ for homogeneously broadened lines. Clearly, the experimental results are in agreement with theory at high values of $\omega_{\mathrm{e}}$, with or without inhomogeneous broadening.

From the fit values of the absorption amplitudes and line widths, $V_{\mathrm{PP}}\left(M_{\mathrm{I}}\right)$ and $\Delta H_{\mathrm{pp}}^{\mathrm{L}}\left(\omega_{\mathrm{ex}}\right)_{M_{\mathrm{I}}}$, the doubly integrated intensities were calculated from eq 17. Figure 9 shows the variation of the doubly integrated intensity ratios $I_{-1} / I_{0}$ and $I_{+1} / I_{0}$ versus $<B>/ A_{0}$. The solid line and the solid circles that appear to fall on top of it were found from fits of eq 8 to spectra computed from eq 1 using homogeneous and inhomogeneously broadened lines, respectively. The dashed horizontal line is the prediction from perturbation theory.

Figure 10 shows the variation of $A_{\mathrm{abs}} / A_{0}$ vs $<B>/ A_{0}$ for experimental and theoretical spectra. The solid line and the solid circles are theoretical values for homogeneous and inhomogeneously broadened lines, respectively. The dashed line is the perturbation prediction, eq 16 . Figure $10 \mathrm{~b}$ details results at low values of $\left\langle B>/ A_{0}\right.$ demonstrating that the experimental results depart from the theoretical results by a term that is linear in the variable $<B>/ A_{0}$. Interestingly, the perturbation theory, eq 16 follows the rigorous theory with rather high precision even to very high values of $\left\langle B>/ A_{0}\right.$. Of further interest is the fact that adding inhomogeneous broadening to the theoretically generated
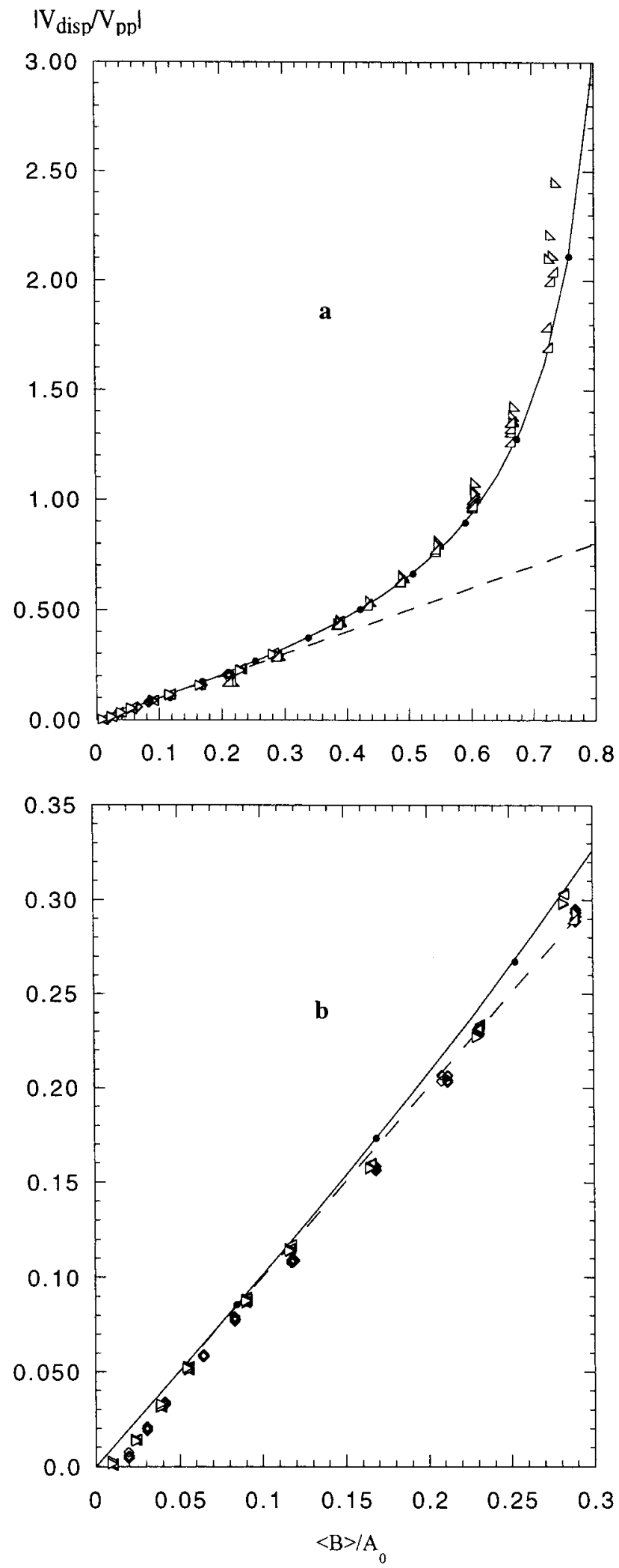

Figure 8. (a) $\left|V_{\text {disp }} / V_{\mathrm{pp}}\right|$ versus $<B>/ A_{0}$ : $16 \mathrm{DSE}$ in EtOH varying [16DSE] at $6.4^{\circ} \mathrm{C}$, for both low- and high-field lines, (diamonds); varying [16DSE] at $25^{\circ} \mathrm{C}$, low-, (right isosceles triangles) and highfield (left isosceles triangles) lines; varying the temperature for 51.7 $\mathrm{mM}$ 16DSE, low-, (right right triangles) and high-field (left right triangles) lines. The solid line is derived from the rigorous theory, eq 1. The solid circles that appear to fall on top of the solid line are computed from eq 1 using inhomogeneously broadened lines. The dashed line is the prediction of the perturbation theory. (b) is an expanded presentation of (a) at lower values of spin exchange frequency. The theoretical curves have no adjustable parameters. 


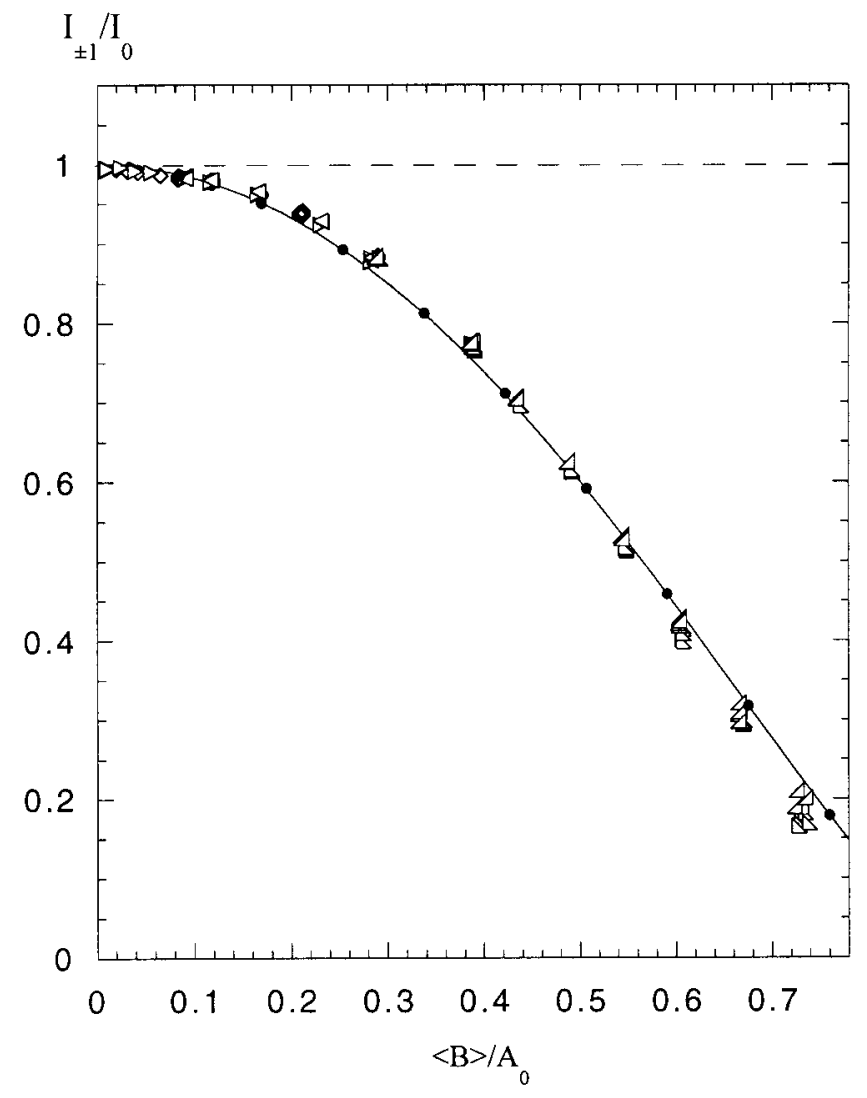

Figure 9. Doubly integrated intensity ratios of the outer lines to the central line, $I_{-1} / I_{0}$ and $I_{+1} / I_{0}$ versus $\left\langle B>/ A_{0}\right.$ plotted using the same symbols as in Figure 8 (a). The solid line and the solid circles that appear to fall on top of them were found from eq 1 using homogeneous and inhomogeneously broadened lines, respectively. The dashed horizontal line is the prediction from perturbation theory. The theoretical curves have no adjustable parameters.

spectrum in eq 1 leaves the predicted variation of $A_{\mathrm{abs}} / A_{0}$ vs $<B>/ A_{0}$ unchanged as is evidenced by the coincidence of the solid circles with the solid line. It's important to recognize that the discrepancy in Figure 10 is not due to a failing of the theory at high values of $\omega_{\mathrm{e}}$; the discrepancy occurs at low values of $\omega_{\mathrm{e}}$ in the region affected the most by unresolved hyperfine structure. In fact, at higher values of $\omega_{\mathrm{e}}$, Figure 10 gives the impression that the rigorous and perturbation theories begin to parallel the experimental results.

There is, in principle, an effect on the values of $A_{\mathrm{abs}}$ due to small changes in sample polarity as 16DSE is added to EtOH. The magnitude of this effect can be estimated employing the nonempirical polarity index, $\mathrm{H}$, introduced by Mukerjee et al. ${ }^{15}$ which is defined to be the ratio of molar concentration of $\mathrm{OH}$ dipoles in a solvent or solvent mixture to that in water. Assuming ideal mixing as a first order approximation, it is easily shown that the change in $\mathrm{H}$ in going from zero to $52 \mathrm{mM}$ 16DSE is approximately $8 \times 10^{-3}$. From previous work(eq 12 of ref 4 ), it is known that this change in $H$ corresponds to a change in the nitrogen hyperfine coupling constant of approximately 11 $\mathrm{mG}$, or a change in $A_{\mathrm{abs}} / A_{0}$ of about $8 \times 10^{-4}$. This is the maximum effect anticipated for the most concentrated sample and corresponds only to about twice the size of the symbols in Figure 10 and is therefore a negligible effect.

The difference in the behavior of the line shifts, $A_{\mathrm{abs}} / A_{0}$ vs $<B>/ A_{0}$, for PADS, which follows theory extremely well ${ }^{1}$, and for 16DSE which does not, suggests that the unresolved hyperfine structure is responsible for the failing of the theory in the latter case.
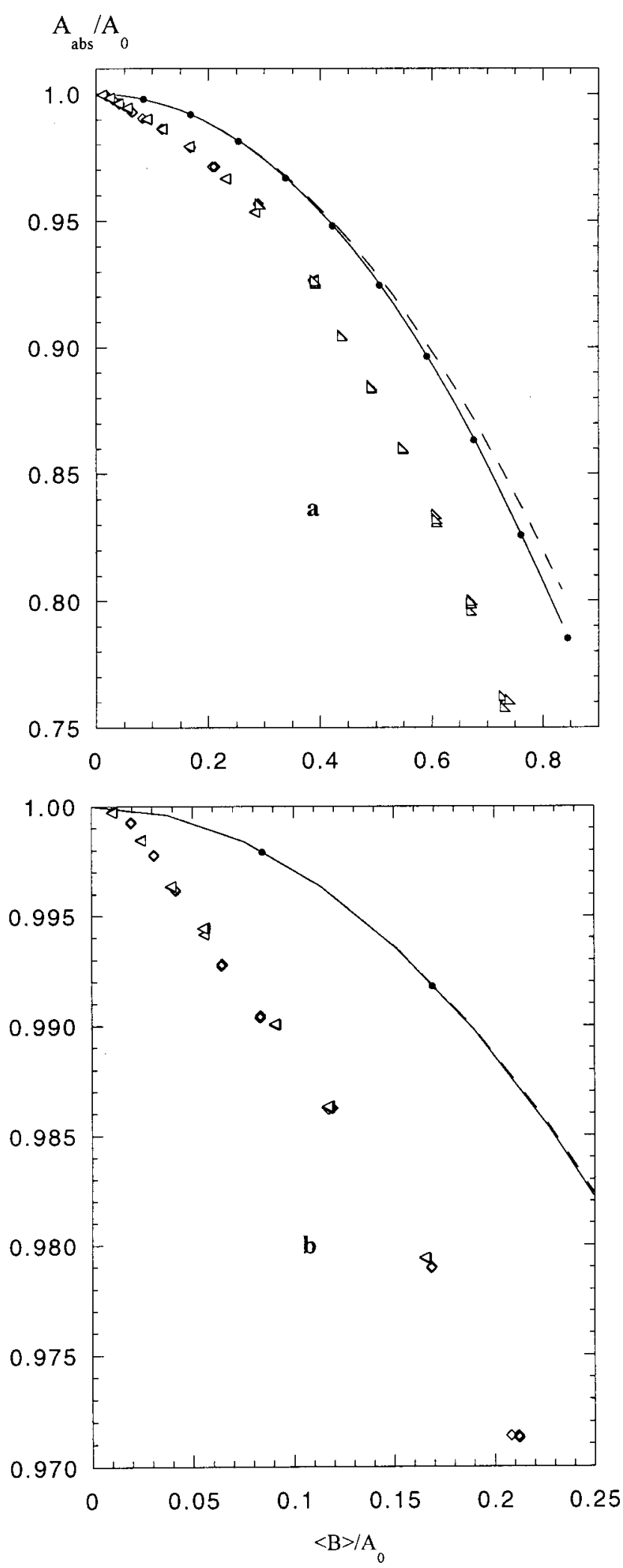

Figure 10. (a) Normalized hyperfine spacing $A_{\text {abs }} / A_{0}$ versus $<B>/ A_{0}$ : varying [16DSE] at $6.4{ }^{\circ} \mathrm{C}$, (diamonds); varying [16DSE] at $25^{\circ} \mathrm{C}$, (left isosceles triangles) varying temperature for $51.7 \mathrm{mM}$ 16DSE, (right right triangles). The solid line and the solid circles that appear to fall on top of it were computed from eq 1 using homogeneous and inhomogeneously broaden lines, respectively. The dashed line is the prediction from perturbation theory, eq 16.

Most experiments would be conducted at spin exchange frequencies well below those producing Figures 4 and 5. For these experiments, the quantity $A_{\mathrm{obs}} / A_{0}$ would normally be 
studied instead of $A_{\mathrm{abs}} / A_{0}$, because it is more sensitive to spin exchange ${ }^{1}$. Keeping in mind that the shift $A_{\text {obs }} / A_{0}$ is due to the shift $A_{\text {abs }} / A_{0}$ plus an additional shift due the overlap of the dispersion lines, it is obvious that $A_{\mathrm{obs}} / A_{0}$ is not in agreement with theory either. For either shift, it would be a simple matter to fit the curves in Figure 10 thereby arriving at an empirical method to determine the spin exchange frequency from the shift. We do not yet offer such an empirical formulation until further work can clarify the dependence of the discrepancy on the unresolved hyperfine pattern and on the solvent.

Figures 8-10 present representative data from this study. All data for temperatures above $-14{ }^{\circ} \mathrm{C}$ fall on these same curves whether the temperature or the concentration are varied. It follows that the broadening is due to spin exchange only, because broadening due to dipolar interactions would not lead to the shifts and intensity changes inherent in eq 1 . A few spectra taken at temperatures down to $-25{ }^{\circ} \mathrm{C}$ gave data departing from the common curves in Figures $8-10$ attributable to the onset of dipolar broadening.

\section{Conclusions}

Spin exchange broadens ${ }^{14} \mathrm{~N}$ spectra according to eq 3 at all values of $\omega_{\mathrm{e}}$, experimentally and theoretically with or without unresolved hyperfine structure. The outer lines broaden faster than the central line with experiment in full accord with the rigorous theory but not with the perturbation theory. For an inhomogeneously broadened line, the broadening must be extracted from the Lorentzian component of the Voigt shape at low values of $\omega_{\mathrm{e}}$.

Numerically, the rigorous theory, eq 1 , is equivalent to three absorption and two dispersion lines as expressed by eq 8 which may mean that the sum in eq 1 could be performed in such a way as to arrive at eq 8 . The fact that the theory can be expressed by eq 8 has the obvious practical advantage of simplifying the least-squares fitting procedures to analyze experimental data. It also provides a basis to study the effect of spin exchange on each of the lines separately. In addition to broadening and shifting the lines, spin exchange reduces the intensity of the outer lines relative to the central line and induces dispersion lines of opposite sign that become a major component of the line shape at high values of $\omega_{\mathrm{e}}$.

The rigorous theory predicts every aspect of the experimentally observed results, except for the shifts $A_{\text {abs }} / A_{0}$, Figure 10 .

Acknowledgment. This research was supported by grants from the NIH S06 GM48680-08 (to BB), NIH 3 R15 GM/ RR494737-01S1 (to M.P.), and grants from the CSUN Research and Grants Committee(to BB and M.P.).

\section{References and Notes}

(1) Bales, B. L.; Peric, M. J. Phys. Chem. B 1997, 101, 8707.

(2) Molin, Y. N.; Salikhov, K. M.; Zamaraev, K. I. Spin Exchange. Principles and Applications in Chemistry and Biology; Springer-Verlag: New York, 1980.

(3) Bales, B. L. In Biological Magnetic Resonance; L. J. Berliner, L. J.; Reuben, J., Ed.; Plenum Publishing Corporation: New York, 1989; p 77.

(4) Bales, B. L.; Howe, A. M.; Pitt, A. R.; Roe, J. A.; Griffiths, P. C. J. Phys. Chem. B 2000, 104, 264.

(5) Bales, B. L.; Ranaganathan, R.; Griffiths, P. C. J. Phys. Chem. B 2001, 105, 7465 .

(6) Bales, B. L.; Mareno, D.; Harris, F. L. J. Magn. Reson. A 1993 $104,37$.

(7) Currin, J. D. Phys. Rev. 1962, 126, 1995.

(8) Halpern, H. J.; Peric, M.; Yu, C.; Bales, B. L. J. Magn. Reson. 1993, 103, 13.

(9) Eastman, M. P.; Bruno, G. V.; Freed, J. H. J. Chem. Phys. 1970, $52,2511$.

(10) Jost, P.; Griffith, O. H. In Spin Labeling. Theory and Applications.;

Berliner, L. J., Ed.; Academic Press: New York, 1976; p 251.

(11) Fox, K. K. J. Chem. Soc., Faraday Trans. 1 1978, 74, 220.

(12) Bales, B. L.; Wajnberg, E.; Nascimento, O. R. J. Magn. Reson 1996, A 118,227

(13) Bales, B. L.; Peric, M.; Lamy-Freund, M. T. J. Magn. Reson. 1998, 132, 279.

(14) Fraenkel, G. K. J. Chem. Phys. 1965, 42, 4275

(15) Mukerjee, P.; Ramachandran, C.; Pyter, R. A. J. Phys. Chem. 1982 86,3189 . 